\title{
Optimal Surface Parameterization Using Inverse Curvature Map
}

\author{
Yong-Liang Yang, Junho Kim, Feng Luo, Shi-Min Hu, Member, IEEE, and Xianfeng Gu, Member, IEEE
}

\begin{abstract}
Mesh parameterization is a fundamental technique in computer graphics. The major goals during mesh parameterization are to minimize both the angle distortion and the area distortion. Angle distortion can be eliminated by the use of conformal mapping, in principle. Our paper focuses on solving the problem of finding the best discrete conformal mapping that also minimizes area distortion. First, we deduce an exact analytical differential formula to represent area distortion by curvature change in the discrete conformal mapping, giving a dynamic Poisson equation. On a mesh, the vertex curvature is related to edge lengths by the curvature map. Our result shows the map is invertible, i.e., the edge lengths can be computed from the curvature (by integration). Furthermore, we give the explicit Jacobi matrix of the inverse curvature map. Second, we formulate the task of computing conformal parameterizations with least area distortions as a constrained nonlinear optimization problem in curvature space. We deduce explicit conditions for the optima. Third, we give an energy form to measure the area distortions, and show that it has a unique global minimum. We use this to design an efficient algorithm, called free boundary curvature diffusion, which is guaranteed to converge to the global minimum; it has a natural physical interpretation. This result proves the common belief that optimal parameterization with least area distortion has a unique solution and can be achieved by free boundary conformal mapping. Major theoretical results and practical algorithms are presented for optimal parameterization based on the inverse curvature map. Comparisons are conducted with existing methods and using different energies. Novel parameterization applications are also introduced. The theoretical framework of the inverse curvature map can be applied to further study discrete conformal mappings.
\end{abstract}

Index Terms-Mesh, conformal parameterization, poisson, metric, curvature, inverse map.

\section{INTRODUCTION}

CuRface parameterization is the process of mapping a Surface to a planar region, and it has broad applications in graphics. Parameterizations introduce distortions between the original surface and its planar image, which can be separated into angle distortion and area distortion [1], [2], [3]. In theory, angle distortion can be eliminated completely by conformal mapping, but it is impossible for conformal mappings to further eliminate area distortion completely, except for developable surfaces.

For a given surface, we can define infinitely many different conformal mappings with different area distortions, as shown in Fig. 1. The central problem of the optimal parameterization can be stated as follows:

How can we find the best conformal mapping that has the least area distortion?

In this paper, we present a set of theoretical tools as well as practical algorithms to tackle this problem.

- Y.-L. Yang and S.-M. Hu are with the Department of Computer Science and Technology, Tsinghua University, Beijing 100084, P.R. China. E-mail:yangyl@co.cs.tsinghua.edu.cn, shimin@tsinghua.edu.cn.

- J. Kim is with Stony Brook University, Stony Brook, NY 11794-4400 and the College of Visual Image and Information Technology, Dong-Eui University, Room 506, 995 Eom-Gwang Roh, Busan-Jin Gu, Busan 614-714, Korea.E-mail: kim.junho@deu.ac.kr.

- F. Luo is with the Department of Mathematics, Rutgers University, Hill Center-Busch Campus, 110 Frelinghuysen Road, Piscataway, NJ 08854. E-mail: fluo@math.rutgers.edu.

- X. Gu is with Stony Brook University, Room 2425, Computer Science Bldg., Stony Brook, NY 11794-4400. E-mail: gu@cs.sunysb.edu.

Manuscript received 26 Sept. 2007; revised 14 Feb. 2008; accepted 25 Feb. 2008; published online 14 Mar. 2008.

Recommended for acceptance by G. Taubin.

For information on obtaining reprints of this article, please send e-mail to: tvcg@computer.org, and reference IEEECS Log Number TVCG-2007-09-0146. Digital Object Identifier no. 10.1109/TVCG.2008.54.

\subsection{Background}

Parameterization methods have become a fundamental tool in graphics, and a significant amount of research has focused on it. Here, we briefly overview the most related works and refer readers to [1], [2], and [3] for wider surveys.

A common approach for parameterization is to minimize a certain energy to control the distortion. Lévy et al. [4] defined an energy to approximate the Cauchy-Riemann equation; Desbrun et al. [5] optimized Dirichlet energy. Variations of harmonic energies were also optimized using discrete Laplace-Beltrami operators in [6], [7], [8], [9], [10], and [11]. More general energy forms can be found in [12], [13], [14], [15], [16], and [17]. Most linear methods apply a convex Dirichlet-type boundary. Virtual boundaries were applied in [17] and [18] to absorb distortions introduced by the convex boundary conditions. Alternatively, [4] and [5] provided parameterizations which require to fix only a few vertices in the parametric domain. Karni et al. [19] discussed the design of geometrically complex boundary conditions with constraints. Zayer et al. [20] applied discrete tensorial quasiharmonic maps to improve the boundary and reduce the distortion.

One of the most prominent characteristics of conformal mapping is that it preserves angles. Angle-based flattening $(\mathrm{ABF})$ method [21] utilizes this property to produce highquality conformal mappings. It derives the discrete conformal mapping by minimizing the ABF energy which is defined as differences between the corner angles of faces on the original mesh and their images on the parameter plane. During the process, the boundary evolves freely to further reduce the distortion. Recently, the method has been improved by several derivative works [22], [23] in terms of speed and robustness. 


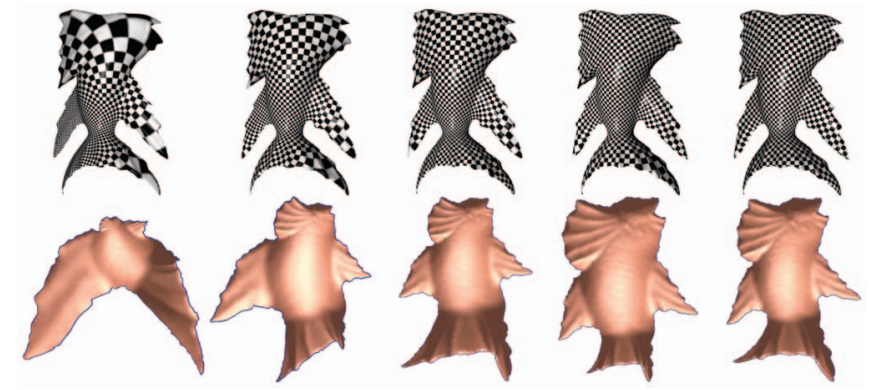

Fig. 1. There are an infinity number of conformal parameterizations for a given surface. We minimize the area distortion within the conformal mappings.

Another characteristic of conformal mapping is that it can map infinitesimal circles to infinitesimal circles and preserve their intersection angles. This inspired the circle packing method in [24]. Circle packings and circle patterns replace infinitesimal circles with finite circles. In the limit of refinement, the continuous conformal maps are recovered [25]. Collins and Stephenson [26] have implemented circle packing in their software CirclePack which only considers combinatorics. The connection between circle packing and smooth surface Ricci flow [27] was discovered in [28]. The discrete Ricci flow method was introduced in [29] for hyperbolic parameterization.

Kharevych et al. [30] provided conformal parameterizations for arbitrary genus types by applying circle patterns based on the variational principle in Bobenko and Springborn [31]. The method in [30] supports very flexible boundary conditions ranging from free boundaries to control of the boundary shape via prescribed curvatures. It can further reduce the distortion by incorporating manually selected cone singularities. Ben-Chen et al. [32] introduced a conformal parameterization which automatically determines the locations and target curvatures of the cone singularities.

Our work differs from the previous works in the following aspects. Based on a rigorous theoretic proof, our method can produce a discrete conformal parameterization with least area distortion among all possible parameterizations. Similar to [30], our method is applicable for meshes with general topologies. Furthermore, the method can be extended for optimizing more general energies with constraints on curvatures and area distortions. (Fig. 8 shows parameterizations with special curvature constraints, such that all boundary curvatures are constant.)

In this paper, we will explain our theoretical results and algorithmic implementations with circle packings. Since circle packing and circle patterns are equivalent in theory [33], [34], our results can also be explained with the setting of circle patterns (see Appendix B).

\subsection{Overview}

Most of the previous works minimize some energy forms which measure both angle distortion and area distortion. In this work, we take the approach similar to those in [5] and [20] to separate these two criteria. As shown in Fig. 1, we only minimize the area distortion within the conformal mappings, which eliminate the angle distortions.

We address the angle distortion by using the discrete conformal mappings based on circle packing. The given mesh is covered by circles, each of which is centered at a vertex as shown in Fig. 3. A circle centered at a vertex is tangent to or intersects with another circle centered at its neighbor vertex. We approximate the conformal mapping by varying the radii while preserving the intersection angles among the circles (see Section 2).

With circle packing, we can establish the mapping from the configuration of radii to the configuration of curvatures, the so-called curvature map $K$ :

$$
\begin{aligned}
K: & \{\text { configuration of radii }\} \\
& \rightarrow\{\text { configuration of curvatures }\} .
\end{aligned}
$$

We show that the curvature map is bijective in the conformal mapping. We give an analytical formula for the inverse curvature map by explicitly computing its Jacobian, which is revealed as a dynamic Poisson equation (see Section 2). Therefore, we can easily compute the radii from the prescribed curvatures.

Discrete conformal parameterization can be treated as finding a configuration of radii such that all curvatures are zeros except for those at the boundaries and cone singularities. All curvature configurations corresponding to parameterizations form an affine subspace, which we call the admissible curvature space. Area distortions can be measured by various energy forms defined on the configurations of radii. Optimal conformal parameterization is equivalent to minimizing the specific energy in the admissible curvature space, and therefore, it is a nonlinear optimization problem with linear constraints.

Energies with good properties, such as differentiability, unique global minimum, simple forms of gradient, and Hessian, are highly preferred in practice. We discovered an energy form that met all the requirements (see Section 3.5). Furthermore, a simple curvature flow algorithm with free boundary conditions is guaranteed to converge to the global minimum.

The pipeline of optimal parameterization system is as follows:

1. mesh preparation (Section 3.1),

2. computing the initial circle packing metric (Section 3.2),

3. selecting the singular vertex set (Section 3.3),

4. computing the optimal circle packing metric (Sections 3.4 and 3.5), and

5. isometric embedding (Section 3.6).

The theoretical results of inverse curvature map are explained in Section 2. Each step of the algorithm pipeline is elucidated in Section 3. The experimental results are demonstrated in Section 4. We conclude our work and point out the future direction in Section 5. Detailed theoretical proofs are presented in the Appendix.

\section{Inverse Discrete Curvature Map}

In this section, we introduce the inverse curvature map, which is the key ingredient of our optimal parameterization.

The discussion is based on general triangular meshes with arbitrary topologies. We denote a mesh by $M=\{V, E, F\}$. A vertex, an edge, and a face are denoted as $v_{i},\left[v_{i}, v_{j}\right]$ (or $\left.e_{i j}\right)$, and $\left[v_{i}, v_{j}, v_{k}\right]$ (or $f_{i j k}$ ), respectively. A mesh $M$ embedded in $\mathbb{R}^{3}$ has a naturally induced euclidean metric, which is determined by each edge length. The vertex curvatures are defined as follows: For an interior vertex, the curvature equals $2 \pi$ minus the sum of angles between edges at the vertex, whereas 
for a boundary vertex, it is $\pi$ minus this sum. The discrete Gauss-Bonnet theorem states that the total curvature is $2 \pi \chi(M)$, where $\chi(M)$ is the Euler number of the mesh.

\subsection{Circle Packing Metric}

Given a triangular mesh, we associate to each vertex $v_{i}$ a circle with radius $\gamma_{i}$. On edge $e_{i j}$, the two circles with radii $\gamma_{i}$ and $\gamma_{j}$ intersect at an angle of $\phi_{i j}$, as shown in Fig. 3.

Definition 2.1 (circle packing metric). A circle packing metric for a mesh $M$ is defined as $(M, \Gamma, \Phi)$, where $M$ represents the triangulation, $\Gamma: V \rightarrow \mathbb{R}^{+}$is the circle radius function for vertices (i.e., $v_{i} \mapsto \gamma_{i}$ ), and $\Phi: E \rightarrow\left[0, \frac{\pi}{2}\right]$ is the angle function for edges (i.e., $e_{i j} \mapsto \phi_{i j}$ ). The discrete metric on an edge $e_{i j}$ is determined by $l_{i j}=\sqrt{\gamma_{i}^{2}+\gamma_{j}^{2}+2 \gamma_{i} \gamma_{j} \cos \phi_{i j}}$.

Now, the edge lengths can be determined by the circle radii $\gamma_{i}$ and the intersection angles $\phi_{i j}$ with the cosine law, depicted in Fig. 3. Since the edge lengths determine the angles on each face, the circle radii determine the vertex curvatures. We designate the mapping from the configuration of radii to the configuration of the vertex curvatures as the curvature map.

\subsection{Inverse Curvature Map}

Two circle packing metrics of the same mesh $M,\left(M, \Gamma_{1}, \Phi_{1}\right)$ and $\left(M, \Gamma_{2}, \Phi_{2}\right)$, are conformal to each other, if $\Phi_{1}$ equals $\Phi_{2}$. Each conformal equivalence class of circle packing metrics forms a space which we call a conformal discrete metric space, denoted by $U$. Upon fixing the edge angles $\phi_{i j}$, a discrete circle packing metric can be represented by a vector $\mathbf{u}=\left(u_{1}, \cdots, u_{n}\right)$, where $u_{i}=\log \gamma_{i}, u_{i} \in(-\infty,+\infty)$, and $n$ is the number of vertices. Each conformal discrete metric space is homeomorphic to $\mathbb{R}^{n}$. Because scaling does not affect the curvature, we normalize the conformal metrics by requiring $\sum_{i} u_{i}=0$, which defines a hyperplane in the $\mathbb{R}^{n}$ that we denote $\Pi_{\mathbf{u}}$. The discrete curvature $K$ maps each $\mathbf{u}$ to a curvature function $\mathbf{k}=\left(k_{1}, k_{2}, \cdots, k_{n}\right)$, and the image of $\Omega_{\mathrm{k}}:=K\left(\Pi_{\mathrm{u}}\right)$ is a convex polytope [28].

The curvature map $K$ from the conformal metric space to the curvature space $K: \Pi_{\mathrm{u}} \rightarrow \Omega_{\mathrm{k}}$ is bijective; both the map and the inverse map have an infinite degree of smoothness. Furthermore, the curvature map is real analytic (so it can be represented as the summation of an infinite series).

Theorem 2.2 (inverse curvature map). The curvature map $K$ from a conformal class of circle packing metrics $\Pi_{\mathbf{u}}$ to the curvature space $\Omega_{\mathrm{k}}$ is a $C^{\infty}$ diffeomorphism. Furthermore, it is real analytic.

The derivative map $d K: T \Pi_{\mathbf{u}}(\mathbf{u}) \rightarrow T \Omega_{\mathbf{k}}(\mathbf{k})$ satisfies the discrete Poisson equation

$$
d \mathbf{k}=\Delta(\mathbf{u}) d \mathbf{u},
$$

where $T \Pi_{\mathbf{u}}(\mathbf{u})$ is the tangent space of $\Pi_{\mathbf{u}}$ at the point $\mathbf{u}$, $T \Omega_{\mathbf{k}}(\mathbf{k})$ is the tangent space of $\Omega_{\mathbf{k}}$ at the point $\mathbf{k}$, and $\Delta(\mathbf{u})$ is a positive definite matrix when restricted to $T \Pi_{\mathbf{u}}(\mathbf{u})$.

Therefore, the curvature map and the inverse curvature map can be represented as

$$
\mathbf{k}_{1}-\mathbf{k}_{0}=\int_{\mathbf{u}_{0}}^{\mathbf{u}_{1}} \Delta(\mu) d \mu, \quad \mathbf{u}_{1}-\mathbf{u}_{0}=\int_{\mathbf{k}_{0}}^{\mathbf{k}_{1}} \Delta(\xi)^{-1} d \xi
$$

A detailed proof can be found in Appendix A. Here, we give an intuitive picture using a differential network flow model as shown in Fig. 4. We treat the mesh as a network. Curvature flows along the edges when vertex radii change. Suppose $v_{i}$ and $v_{j}$ are two adjacent vertices, such that the logarithms of the radii change by $\delta u_{i}$ and $\delta u_{j}$, respectively, and the conductivity (weight) for the edge is $w_{i j}>0$, which depends on the current vertex radii.

Then, the curvature flux from $v_{i}$ to $v_{j}$ along the edge is $\delta k_{i j}=w_{i j}\left(\delta u_{j}-\delta u_{i}\right)$. Each vertex has several edges connected to it, so the net edge curvature flux equals the overall curvature change at the vertex, $\delta k_{j}=\sum_{i} \delta k_{i j}$. Therefore, the Laplace matrix has an explicit form: $\Delta=\left(d_{i j}\right)$

$$
d_{i j}= \begin{cases}-w_{i j} & i \neq j,\left[v_{i}, v_{j}\right] \in E \\ \sum_{k} w_{i k} & i=j, \\ 0 & i \neq j,\left[v_{i}, v_{j}\right] \notin E .\end{cases}
$$

We now explain the geometric meaning of the edge weight. On each face, there exists a unique circle perpendicular to all three circles, as shown in Fig. 3 with the red circle. The center of the circle is the radial center (or power center). Then, the weight for a halfedge equals to $\frac{h_{i j}}{l_{i j}}$, where $h_{i j}$ is the distance from the radial center to the halfedge, and $l_{i j}$ is the current length of the halfedge. The edge weight is the sum of its two halfedge weights and depends on the current curvature (or, equivalently, the radii). Therefore, the Laplace-Beltrami operator is dynamic. This fact makes the whole theory more complicated.

Algorithm 1 computes the inverse curvature map, which can be applied for conformally parameterizing general meshes directly. Fig. 5 demonstrates some parameterization results using this algorithm.

Algorithm 1 for computing the discrete conformal metric $\overline{\mathbf{u}}$ from the prescribed curvature $\overline{\mathbf{k}}$ is as follows:

Algorithm 1: Inverse curvature map $\overline{\mathbf{u}}=K^{-1}(\overline{\mathbf{k}})$

Compute the initial circle packing metric $(M, \Gamma, \Phi)$

Compute initial curvature $\mathbf{k}$

$\mathbf{u} \Leftarrow \mathbf{u}_{0}$, where $\mathbf{u}_{0}$ is the initial circle packing metric.

while $|\overline{\mathbf{k}}-\mathbf{k}|>\varepsilon$ do

Compute $w_{i j}(\mathbf{u})$ to form the Laplace matrix $\Delta(\mathbf{u})$.

$d \mathbf{u} \Leftarrow \Delta(\mathbf{u})^{-1}(\overline{\mathbf{k}}-\mathbf{k})$

$\mathbf{u} \Leftarrow \mathbf{u}+d \mathbf{u}$

$\mathbf{k} \Leftarrow K(\mathbf{u})$

end while

$\overline{\mathbf{u}} \Leftarrow \mathbf{u}$

\subsection{Relation with Discrete Ricci Flow}

Inverse curvature map can also be deduced from the theory of discrete Ricci flow [28]. The fact that curvature map $K$ : $\mathbf{u} \rightarrow \mathbf{k}$ is invertible is proved in the following way.

Let $\mathbf{u}_{0}$ be the initial metric with the curvature $\mathbf{k}_{0}$. Suppose $\overline{\mathbf{k}}$ is the prescribed curvature and its corresponding metric is $\overline{\mathbf{u}}$, then we can define the following discrete Ricci energy as

$$
E_{R i c}(\mathbf{u})=\int_{\mathbf{u}_{0}}^{\mathbf{u}}(\overline{\mathbf{k}}-\mathbf{k})^{T} d \mu .
$$




Mesh Preparation $\rightarrow$ Compute the Initial CP Metric $\rightarrow$ Select the Singular Vertex Set $\rightarrow$ Optimization $\rightarrow$ Embedding

Fig. 2. Algorithm pipeline.

From discrete Ricci flow theory, $E_{R i c}(\mathbf{u})$ is convex in the subaffine space $\sum u_{i}=0$, and $\overline{\mathbf{u}}$ is the unique global minimal point. The target metric $\overline{\mathbf{u}}$ can be obtained by minimizing the energy, using the steepest descent method

$$
\frac{d \mathbf{u}}{d t}=-\nabla E_{R i c}(\mathbf{u})=-(\overline{\mathbf{k}}-\mathbf{k})
$$

which is the discrete Ricci flow. Therefore, the curvature map $K$ is invertible.

In order to compute the optimal parameterization, we need an explicit form of the Jacobi matrix of the curvature map, which is related to the Hessian matrix of $E_{\text {Ric }}(\mathbf{u})$. This is the key step for a nonlinear optimization algorithm.

\section{Optimal Surface Parameterization}

This section explains the algorithm pipeline for the optimization system as shown in Fig. 2. Each section corresponds to one step in Fig. 2, respectively.

\subsection{Mesh Preparation (Optional)}

In practice, the initial circle packing metric requires all the edge angles to be acute, so that the Jacobi matrix of the curvature map is positive definite (equivalently, the Ricci energy (4) is convex) to ensure the uniqueness of the solution. If the input mesh has too many obtuse angles and skinny triangles, we remesh it using the algorithms described in [35] and [36] to improve the mesh quality.

\subsection{Computing the Initial Circle Packing Metric}

We use a simple method for the initial circle packing metric, described in Algorithm 2.
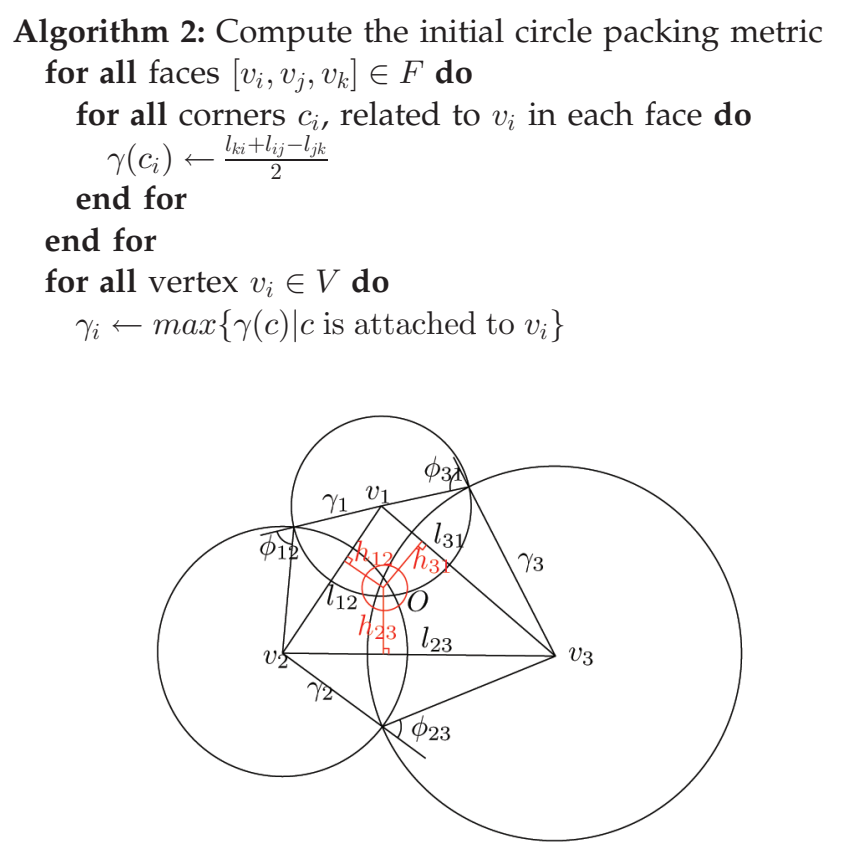

Fig. 3. Circle packing metric. end for

for all edges $\left[v_{i}, v_{j}\right] \in E$ do

$\phi_{i j}=\min \left\{\cos ^{-1} \frac{l_{i j}^{2}-\gamma_{i}^{2}-\gamma_{j}^{2}}{2 \gamma_{i} \gamma_{j}}, \frac{\pi}{2}\right\}$, where $l_{i j}$ is the euclidean

edge length

end for

In experiments, this algorithm guarantees to get acute corner angles, and the initial circle packing metrics are very closed to the induced euclidean metric of the mesh.

\subsection{Selecting Singular Vertex Set}

In order to reduce the area distortion, it is very helpful to concentrate curvatures on a subset of vertices, which we call a singular vertex set. For example, if a mesh has boundaries, all of the boundary vertices are in the singular vertex set in general. Moreover, we can set several interior vertices as singular ones in order to minimize the area distortions. Several effective methods have been introduced to select singular vertices, including manual selection [30], vector field analysis [37], and spectral analysis [32].

We select the singular vertex set in step 3 of algorithm pipeline in Fig. 2. Intuitively, we first let the curvature be uniformly distributed on all vertices, then measure the area distortion, and finally pick the critical points of the area distortion function. The algorithm is described in Algorithm 3. Fig. 6 demonstrates the algorithm using the Stanford Bunny model.

Algorithm 3: Compute the singular vertex set

$\bar{k}_{i} \leftarrow 0$ for $v_{i} \in \partial M$,

$\bar{k}_{i} \leftarrow \frac{2 \pi \chi(M)}{n}$ for $v_{i} \notin \partial M$, where $n$ is number of interior vertices

$\overline{\mathbf{u}} \leftarrow K^{-1}(\overline{\mathbf{k}})$ using Algorithm 1

$S \Leftarrow \partial M \cup\{$ local minima of $\overline{\mathbf{u}}\}$

\subsection{Compute the Optimal Circle Packing Metric}

Now, we explain the process to compute the optimal circle packing metrics in step 4 of the algorithm pipeline in Fig. 2. There are two methods for the optimization: the projected gradient method described in Section 3.4.1 and the free boundary curvature diffusion method described in Section 3.5.

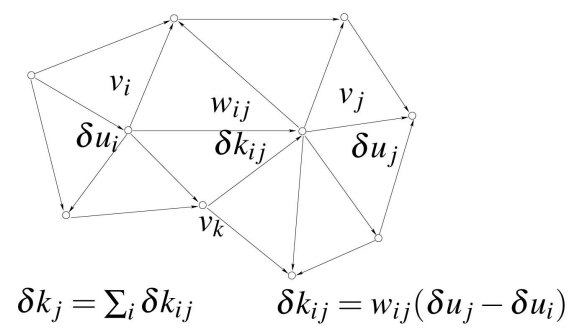

Fig. 4. Differential network curvature flow model. The curvature flux along edge $\delta k_{i j}$ is driven by the gradient of $\delta \mathbf{u}$. The change of curvature at a vertex $\delta k_{i}$ equals the divergence of the curvature flow. 

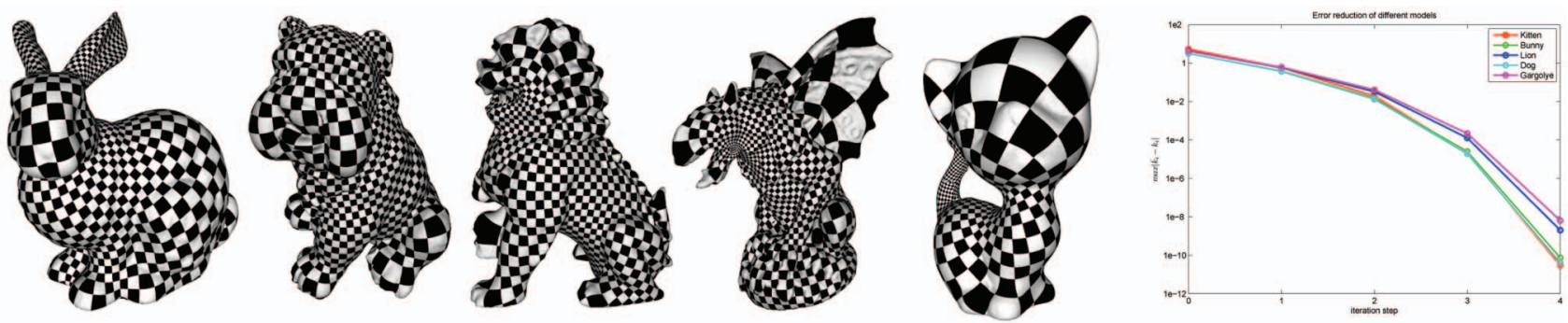

Fig. 5. Discrete conformal parameterizations using inverse curvature map ([\#v, \#f, execution time (in seconds)] are [20,660, 41,118, 6.3], [29,923, $59,417,11.2]$, [20,224, 40,118, 5.8], [20,618, 40,803, 6.6], and [10,219, 20,438, 2.5], respectively, with Pentium 42.8 GHz with 2-GB memory).

\subsubsection{Projected Gradient Method for Optimization}

We can define various energy forms to measure the area distortion. The energy can be defined either in the conformal metric space or in the curvature space. In terms of the computational complexity, they are equivalent. We decide to define the energy in the curvature space because this is a convex affine subspace. If the energy is convex, only one optimum exists. Therefore, it is easy to handle both theoretically and practically.

The possible solutions must be valid parameterizations, namely, all curvatures are zeros except at the singularities.

Definition 3.1 (admissible curvature space). Given a mesh $M$, the vertices are divided into two sets $S$ and $N . S$ represents the singular vertices, whereas $N$ represents nonsingular vertices. The admissible curvature space is an affine subspace defined as the intersection of the following hyperplanes:

$$
\Pi_{\mathbf{k}}:=\bigcap_{v_{i} \in N}\left\{k_{i}=0\right\} \bigcap\left\{\sum_{v_{j} \in S} k_{j}=2 \pi \chi(M)\right\} \bigcap \Omega_{\mathbf{k}} .
$$

Then, the optimal parameterization problem is equivalent to optimizing some energy form $E(\mathbf{k})$ in the admissible curvature space.

Optimal parameterization problem. Compute the minima of the energy $E(\mathbf{k})$ in the admissible curvature space $\Pi_{\mathbf{k}}$ :

$$
\min _{\mathbf{k}} E(\mathbf{k}), \text { s.t. } \mathbf{k} \in \Pi_{\mathbf{k}} .
$$

Basically, we compute the gradient of $E(\mathbf{k})$ with respect to $\mathrm{k}$, denoted as $\nabla_{\mathrm{k}} E$, and project the gradient to the affine subspace $\Pi_{\mathrm{k}}$. While the projected gradient is not equal to zero, $\mathrm{k}$ is updated along its direction. Namely, at a critical
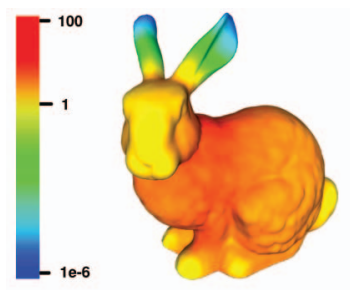

(a)

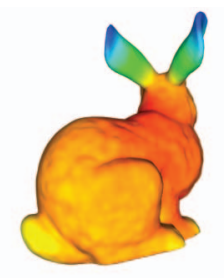

(b)

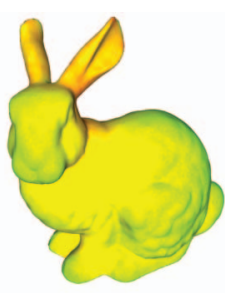

(c)
Fig. 6. Singularity selection process: (a) and (b) depict the area distortion of the bunny model without any singular vertex. We select critical points in ear tips and the point between the roots of the ears. (c) Shows the area distortion after computing the inverse curvature map with the selected singular vertices. The uniformity of the area distortion is greatly improved. point of $E(\mathbf{k}), \nabla_{\mathbf{k}} E$ is orthogonal to the admissible curvature space. This procedure can find one local minimum but does not guarantee that neither all minima, nor the global minimum can be found. In next section, we design a special energy with a unique minimum, in order that this algorithm can reach the global minimum.

Algorithm 4: Optimal discrete conformal parameterization Randomly select a $\mathbf{k} \in \Pi_{\mathbf{k}}$

repeat

$$
\begin{aligned}
& \mathbf{u} \leftarrow K^{-1}(\mathbf{k}) \text { using Algorithm } 1 \\
& \text { Compute the gradient } \nabla_{\mathbf{u}} E \\
& \nabla_{\mathbf{k}} E \Leftarrow \Delta(\mathbf{u})^{-1} \nabla_{\mathbf{u}} E \\
& \text { for all } v_{i} \in N \text { do } \\
& \quad \nabla_{\mathbf{k}} E \Leftarrow \nabla_{\mathbf{k}} E-<\nabla_{\mathbf{k}} E, \mathbf{e}_{i}>\mathbf{e}_{i} \\
& \text { end for } \\
& \nabla_{\mathbf{k}} E \leftarrow \nabla_{\mathbf{k}} E-<\nabla_{\mathbf{k}} E, \mathbf{d}>\frac{\mathbf{d}}{|\mathbf{d}|^{2}} \\
& \mathbf{k} \leftarrow \mathbf{k}-\lambda \nabla_{\mathbf{k}} E \\
& \text { until }\left|\nabla_{\mathbf{k}} E\right|<\varepsilon
\end{aligned}
$$

where $\mathbf{d}$ is a vector with $d_{i}=0$ for nonsingular vertices and $d_{j}=1$ for singular vertices.

Theorem 3.2. Suppose $\mathbf{k}$ is an interior point of $\Pi_{\mathbf{k}}$, also an optimum for an energy form $E(\mathbf{u})$, then all the components of $\nabla_{\mathbf{k}} E$ corresponding to the singular vertices are equal.

Proof. The proof is based on the KKT theorem [38]. If $\mathbf{k}$ is an optimum of $E(\mathbf{k})$, then $\nabla_{\mathbf{k}} E \perp \Pi_{\mathbf{k}}$. Suppose $v_{i}$ is a nonsingular vertex, the normal to the hyperplane $\left\{k_{i}=0\right\}$ is $\mathbf{e}_{i}$, the normal to the plane $\left\{\sum_{v_{j} \in S} k_{j}=\right.$ $2 \pi \chi(M)\}$ is $\mathbf{d}$, where $d_{i}=0$ for nonsingular vertices $v_{i}$ and $d_{j}=1$ for singular vertices $v_{j}$. Therefore

$$
\nabla_{\mathbf{k}} E=\sum_{v_{i} \in N} \lambda_{i} \mathbf{e}_{i}+\mu \mathbf{d},
$$

where $\mu$ is a real number.

The common energy forms used in the literature are

1. ABF energy defined in [21] — this energy measures the differences between the original and the target angles at all the corners (a corner is determined by a face and one vertex adjacent to it):

$$
E_{A B F}(\mathbf{k})=\sum_{\theta}\left(\theta(\mathbf{k})-\theta\left(\mathbf{k}_{0}\right)\right)^{2},
$$

where $\theta\left(\mathbf{k}_{0}\right)^{\prime}$ s are the original corner angles. 
2. Area distortion energy defined in [5]-this energy measures the ratio between the original face area and the face area on the parameter plane:

$$
E_{A D}(\mathbf{k})=\sum_{f}\left(\frac{s_{f}(\mathbf{k})}{s_{f}\left(\mathbf{k}_{0}\right)}-1\right)^{2},
$$

where $s_{f}\left(\mathbf{k}_{0}\right), s_{f}(\mathbf{k})$ are the areas of the face $f$ under the original metric and the target metric. This energy is the most direct measurement for area distortion.

3. $u$-square energy-this energy is just the norm of $\mathbf{u}$, which is a vector with the logarithms of the change of circle radii. Because the mean of $\mathbf{u}$ is zero, this energy can be treated as the variance of $\mathbf{u}$, which is a measurement of the uniformity of $u_{i}$ :

$$
E_{u^{2}}(\mathbf{k})=\left|\mathbf{u}(\mathbf{k})-\mathbf{u}\left(\mathbf{k}_{0}\right)\right|^{2},
$$

where $\mathbf{u}\left(\mathbf{k}_{0}\right)$ is the initial circle packing metric.

The gradient of an energy $E$ with respect to $\mathbf{u}$ is $\nabla_{\mathbf{u}} E$, which is related to $\nabla_{\mathbf{k}} E$ by

$$
\nabla_{\mathbf{k}} E=\Delta^{-1}(\mathbf{u}) \nabla_{\mathbf{u}} E
$$

\subsection{Curvature Diffusion with Free Boundary Conditions}

The energies introduced above are not satisfactory in practice. First, it is unclear whether they have a unique global minimum or not. Second, their gradient has a complicated form, leading to an expensive computation using the projected gradient method.

In this part, we introduce a novel energy, called curvature entropy, which overcomes the shortcomings of other energies. It has a unique global minimum, its gradient has the simplest form $\mathbf{u}$, and it can efficiently be computed with methods other than the projected gradient method.

Definition 3.3 (curvature entropy energy). The entropy energy is

$$
E_{E N}(\mathbf{k})=\int_{\mathbf{k}_{0}}^{\mathbf{k}}\left(\mathbf{u}-\mathbf{u}_{0}\right)^{T} d \boldsymbol{\xi}
$$

where $\mathbf{u}_{0}$ is the initial metric with the curvature $\mathbf{k}_{0}$ defined with the initial mesh.

The integration measures the uniformity of the area distortion function (i.e., conformal factor). The one-form $\mathbf{u}^{T} d \mathbf{k}$ is a closed one-form and, therefore, the integration is path independent. We can choose an arbitrary path from $\mathbf{k}_{0}$ to $\mathrm{k}$ in the curvature space. The curvature entropy energy is closely related to the $u$-square energy defined in (7):

$$
E_{u^{2}}(\mathbf{u})=\int_{\mathbf{u}_{0}}^{\mathbf{u}}\left(\boldsymbol{\mu}-\mathbf{u}_{0}\right)^{T} I d \boldsymbol{\mu},
$$

where $I$ is the identity matrix. If we replace $I$ by $\Delta(\mathbf{u})^{-1}$ in the above formula, we will get the curvature entropy energy

$$
E_{E N}(\mathbf{u})=\int_{\mathbf{u}_{0}}^{\mathbf{u}}\left(\boldsymbol{\mu}-\mathbf{u}_{0}\right)^{T} \Delta(\boldsymbol{\mu})^{-1} d \boldsymbol{\mu}=\int_{\mathbf{k}_{0}}^{\mathbf{k}}\left(\mathbf{u}-\mathbf{u}_{0}\right)^{T} d \xi .
$$

Both $\Delta^{-1}(\mathbf{u})$ and $I$ are positive definite. Therefore, $u$ square energy and curvature entropy energy are equivalent for the purpose of measuring the uniformity of the area distortion $\mathbf{u}$.

This energy can be directly optimized using the projected gradient method. The gradient of the curvature entropy is very simple:

$$
\nabla_{\mathbf{k}} E_{E N}(\mathbf{u})=\mathbf{u}-\mathbf{u}_{0} .
$$

It can also be minimized by the following curvature diffusion method:

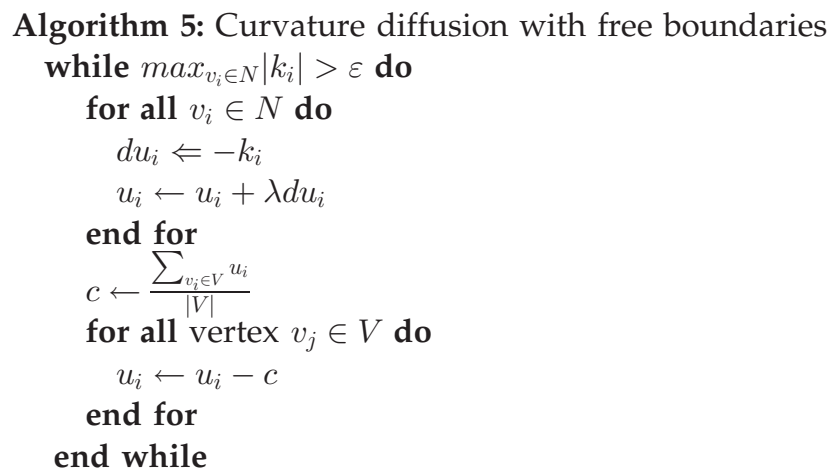

Intuitively, Algorithm 5 sets $\frac{d \mathbf{u}}{d t}=-\mathbf{k}$. According to (1), the curvature will evolve like a heat diffusion, $\frac{d \mathbf{k}}{d t}=-\Delta \mathbf{k}$. The singular vertices absorb all the curvature flux, and the whole surface deforms to be flat in the most natural way. Because the entropy increases in the heat diffusion process, we name this energy curvature entropy.

We show that when the algorithm terminates, the deformation of the singular vertices is uniform.

Lemma 3.4. Suppose $\mathbf{u}$ is the solution of the free boundary curvature diffusion algorithm, then $u_{i}-u_{i}^{0} \equiv$ const, $\forall v_{i} \in S$.

Proof. At the beginning, the $u_{i}-u_{i}^{0}$ terms are zero for all boundary vertices. At each normalization step, $u_{i}-u_{i}^{0}$ changes by the same amount. Therefore, $u_{i}-u_{i}^{0}$ 's are always equal.

The third column in Fig. 7 demonstrates this fact that all the circle radii of the boundary vertices are equal (this is because the radii for all vertices in the initial circle packing metric are equal). This result is consistent with Theorem 3.2, i.e., the gradient of the curvature entropy is $\mathbf{u}$, and this algorithm leads to a solution where all $u_{i}$ s are equal on the singular vertices. Therefore, this algorithm minimizes the entropy in a different approach.

Theorem 3.5. The curvature entropy energy is well defined (namely, the value is independent of the choice of the integration path) and has a unique global minimum point in the admissible curvature space. The free boundary optimization algorithm leads to the global minimum.

Proof. In order to show that the energy is well defined, we need to show that the one-form $\sum\left(u_{i}-u_{i}^{0}\right) d k_{i}$ is closed. Namely, the matrix $\left(\frac{d u_{i}}{d k_{j}}\right)$ is symmetric. $\Delta$ is symmetric, therefore $\Delta^{-1}=\left(\frac{d u_{i}}{d k_{j}}\right)$ is also symmetric.

We can directly compute the gradient of $E_{E N}(\mathbf{k})$, $\nabla_{\mathbf{k}} E_{E N}(\mathbf{k})=\mathbf{u}-\mathbf{u}_{0}$. The necessary condition of the optimum point is $u_{i}-u_{i}^{0}=$ const for all singular vertices.

We further compute the Hessian matrix of $E_{E N}$, which is directly $\Delta^{-1}(\mathbf{k})$. Because $\Delta$ is positive definite, $\Delta^{-1}$ is positive definite. Therefore, $E_{E N}$ is a convex energy. On the other hand, the admissible space of $\mathrm{k}$ is a convex 


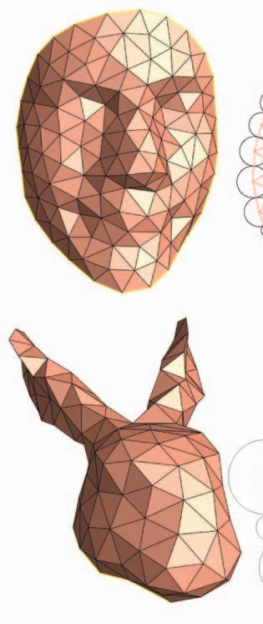

(a)
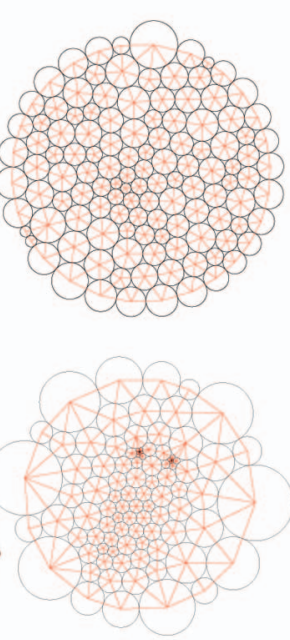

(b)
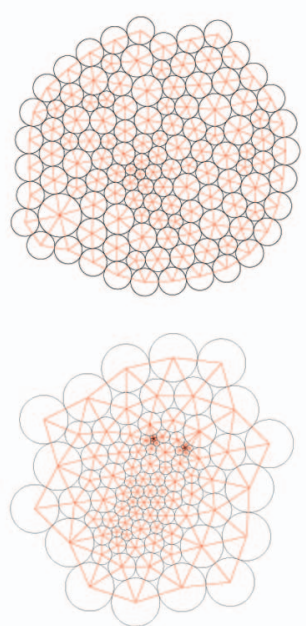

(c)

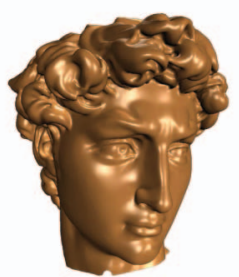

(a)

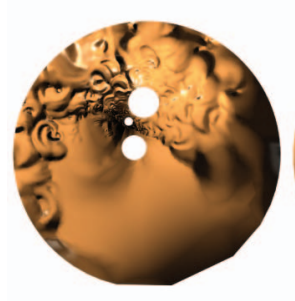

(d)

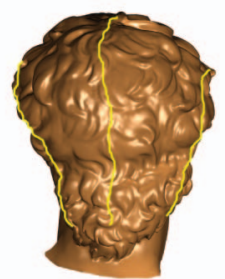

(b)

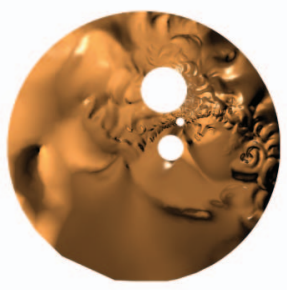

(e)

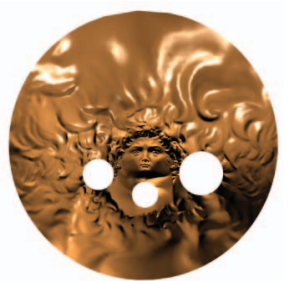

(c)

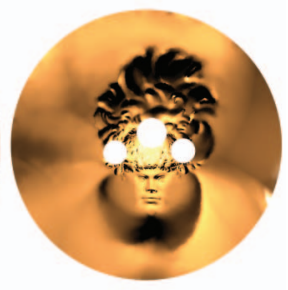

(f)

Fig. 7. Approximating conformal mappings by circle packing. (a) The circle radii are changed, while tangency relations are preserved. (b) Shows a circular boundary condition. (c) Shows a free boundary condition. For the free boundary condition, all circle radii on the boundary vertices are equal.

affine space, implying that the energy has a unique global minimum on it.

The free boundary optimization algorithm can reach one critical point of $E_{E N}$, and $E_{E N}$ has only one unique critical point. Hence, the free boundary curvature optimization algorithm can reach the global minimum.

Comparing to the projected gradient algorithm in Section 3.4.1, the curvature diffusion algorithm does not need to solve the Poisson equation, and it is simple, direct, and easy to implement. With free boundary conditions, we can apply the curvature diffusion algorithm directly.

\subsection{Embedding}

The final step in our algorithm pipeline in Fig. 2 is to isometrically embed the mesh on the plane using the circle packing metric obtained from the optimization.

We first compute a cut on the mesh to slice it to an open topological disk. Several algorithms [39], [40] can be applied directly. Then, we embed the open mesh isometrically onto the plane using the optimal circle packing metric. For meshes with less than $30 k$ faces, we select a face near to the center of the mesh as the root face and directly embed it, then flatten the faces adjacent to it. We propagate the embedding face by face until the whole mesh is flattened.

For large-scale meshes, the propagated errors accumulate, so instead, we use a method similar to [22]. According to Lemma 5.4, the planar embedding $f: M \rightarrow \mathbb{R}^{2}$ is a harmonic map $\Delta f=0$, where the Laplace-Beltrami operator is determined by the final circle packing metric. We first fix the parametric positions of two vertices, then form a linear system to approximate the parameter positions for other vertices in the least squares sense. The result was always found to be a valid embedding for all of our experiments.

\section{Implementations AND EXPERIMENTAL Results}

In this section, we give the experimental results of our algorithms and compare our methods with the state-of-theart techniques including LSCM [4], ABF++ [22], and circle

Fig. 8. Mesh parameterization with the inverse curvature map for a topologically complicated model: The David head model has four boundaries, as shown in (a) and (b). Four different configurations are depicted in (c)-(f), each of which has one outer boundary and three inner boundaries in the parametric space. For each case, the parameterization is obtained by using the inverse curvature map by specifying the sum of target curvatures for an outer boundary as $2 \pi$, the sum of target curvatures for each inner boundary as $-2 \pi$ and $k_{i} e^{-u_{i}}$ is constant for boundary vertices.

patterns [30]. For fair comparisons, we tested the previous methods with the codes which are available on the websites of the original authors.

Table 1 summarizes the statistics of our experiments on several models as shown in Figs. 9, 10, and 11. Angle distortions are measured with three different energy forms: conformality [4], L2 shear [22], and squared sum of angle differences [22]. As shown in Table 1, all of the methods minimize the angle distortion and hold the conformality well.

Area distortions are measured with two different energy forms: L2 stretch in [13] and Log area distortion (8). Our optimization approaches provide the best results for the complicated models, such as the horse and the camel. ABF++ produces small area distortions in many cases, but it may fall into a local minimum (see Fig. 11). The circle patterns provide comparable good results with all the tested models:

$$
\text { Log area distortion }=\sum_{f}\left(\log \frac{s_{f}(\mathbf{k})}{s_{f}\left(\mathbf{k}_{0}\right)}\right)^{2} .
$$

The efficiency of both Algorithm 1 and Algorithm 4 greatly depends on solving the Poisson equation $d \mathbf{k}=\Delta(\mathbf{u}) d \mathbf{u}$. Since the Laplace matrix $\Delta$ is positive definite when restricted on $T \Pi_{\mathbf{u}}(\mathbf{u})$, we use the conjugate gradient method to solve the linear system, which is efficient in terms of time and storage. As shown in Fig. 5, our parameterization with the inverse curvature map is comparable to those of $\mathrm{ABF}++$ and circle pattern methods. The error deduction is very fast as in the convergence chart. Most models can be parameterized within four steps in Algorithm 1.

For optimal parameterization, the free boundary curvature diffusion method (Algorithm 5) is much more efficient than the projected gradient method (Algorithm 4). This is because the latter needs to compute the inverse curvature map for each 
TABLE 1

Comparison of Different Conformal Parameterization Methods

\begin{tabular}{|c|c|c|c|c|c|c|}
\hline Model & Methods & conformality & \begin{tabular}{|c} 
Angle \\
distortion
\end{tabular} & \begin{tabular}{|c|} 
L2 \\
shear
\end{tabular} & $\begin{array}{c}\text { L2 } \\
\text { stretch }\end{array}$ & $\begin{array}{l}\log \text { area } \\
\text { distortion }\end{array}$ \\
\hline camel & LSCM & 0.00002 & 0.00203 & 0.0511 & 185.8090 & 11.84310 \\
\hline \#v: 20775 & $\mathrm{ABF}++$ & 0.00015 & 0.00135 & 0.0453 & 5.1039 & 0.74653 \\
\hline \multirow[t]{5}{*}{ \#f: 40384} & CirclePatterns & 0.00008 & 0.00145 & 0.0472 & 8.5819 & 0.70627 \\
\hline & Free Boundary & 0.00032 & 0.00397 & 0.0699 & 5.1496 & 0.68069 \\
\hline & Optimize U2 & 0.00125 & 0.00374 & 0.0691 & 5.4908 & 1.92130 \\
\hline & Optimize entropy & 0.00019 & 0.00291 & 0.0613 & 5.4141 & 0.74996 \\
\hline & Optimize ABF & 0.00020 & 0.00278 & 0.0596 & 5.4812 & 0.73377 \\
\hline horse & LSCM & 0.00017 & 0.00081 & 0.0310 & 16.8601 & 7.45106 \\
\hline \#v: 31400 & $\mathrm{ABF}++$ & 0.00005 & 0.00047 & 0.0256 & 1.5570 & 0.86409 \\
\hline \multirow{5}{*}{ \#f: 61588} & CirclePatterns & 0.00005 & 0.00046 & 0.0262 & 1.6924 & 0.40167 \\
\hline & Free Boundary & 0.00034 & 0.00195 & 0.0502 & 1.6968 & 0.40979 \\
\hline & Optimize U2 & 0.00051 & 0.00167 & 0.0465 & 1.3928 & 0.36636 \\
\hline & Optimize entropy & 0.00028 & 0.00161 & 0.0458 & 1.7169 & 0.42591 \\
\hline & Optimize ABF & 0.00027 & 0.00156 & 0.0454 & 1.6462 & 0.40199 \\
\hline oliverhand & LSCM & 0.00024 & 0.00034 & 0.0216 & 3.2283 & 3.97385 \\
\hline \#v: 5660 & $\mathrm{ABF}++$ & 0.00007 & 0.00018 & 0.0162 & 1.0274 & 0.05258 \\
\hline \multirow{6}{*}{ \#f: 10782} & CirclePatterns & 0.00012 & 0.00038 & 0.0228 & 1.0278 & 0.05314 \\
\hline & Free Boundary & 0.00117 & 0.00353 & 0.0641 & 1.0383 & 0.06702 \\
\hline & Optimize U2 & 0.00077 & 0.00263 & 0.0569 & 1.0766 & 0.13460 \\
\hline & Optimize entropy & 0.00095 & 0.00264 & 0.0584 & 1.2530 & 0.39787 \\
\hline & Optimize ABF & 0.00081 & 0.00249 & 0.0543 & 1.0363 & 0.06675 \\
\hline & Optimize AD & 0.00104 & 0.00294 & 0.0587 & 57.0863 & 6.40391 \\
\hline woodfish & LSCM & 0.00022 & 0.00036 & 0.0209 & 3.2104 & 3.33530 \\
\hline \#v: 4457 & $\mathrm{ABF}++$ & 0.00008 & 0.00021 & 0.0152 & 1.0126 & 0.02515 \\
\hline \multirow[t]{6}{*}{ \#f: 8449} & CirclePatterns & 0.00008 & 0.00028 & 0.0192 & 1.0135 & 0.02689 \\
\hline & Free Boundary & 0.00162 & 0.00330 & 0.0664 & 1.0171 & 0.02832 \\
\hline & Optimize U2 & 0.00108 & 0.00227 & 0.0571 & 1.0217 & 0.03755 \\
\hline & Optimize entropy & 0.00158 & 0.00315 & 0.0649 & 1.0167 & 0.02803 \\
\hline & Optimize ABF & 0.00128 & 0.00215 & 0.0555 & 1.0167 & 0.03056 \\
\hline & Optimize AD & 0.00066 & 0.00267 & 0.0620 & 3.8291 & 2.09491 \\
\hline
\end{tabular}

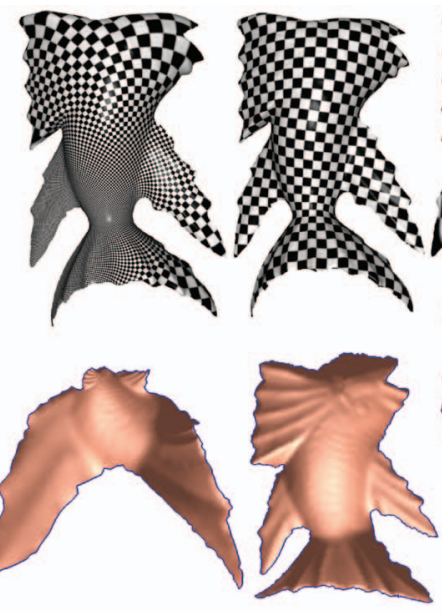

(a)

(b)
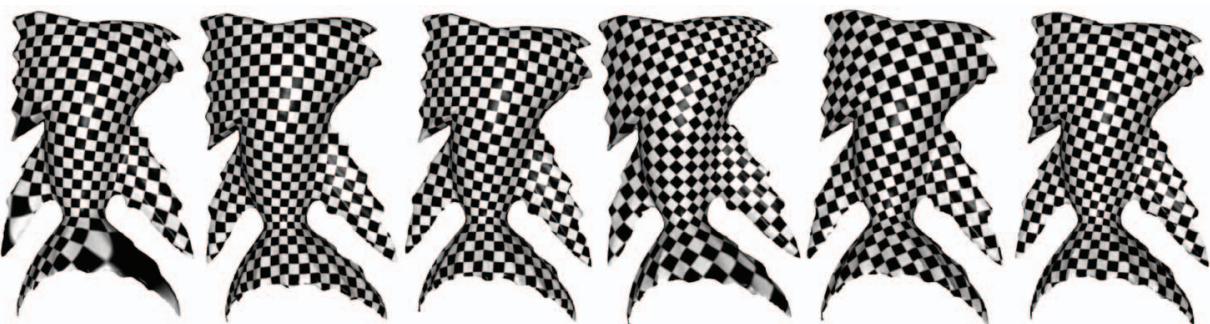

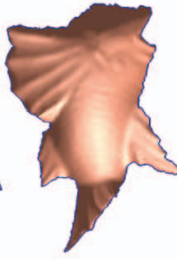

(c)

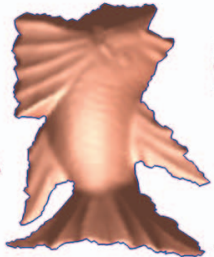

(d)

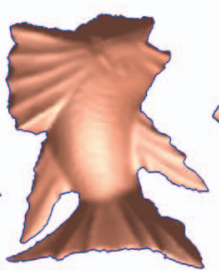

(e)

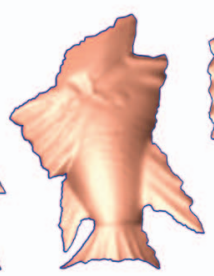

(f)

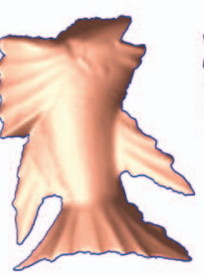

(g)

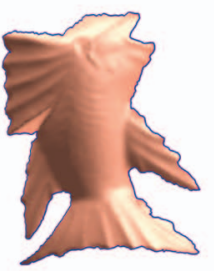

(h)

Fig. 9. Comparison of different parameterization with the Woodfish model: (a) initial, (b), (c), (d), and (e) optimizing ABF, AD, entropy, and U2, respectively, (f), (g), and (h) LSCM, circle patterns, and ABF++, respectively.

iteration step, which is very time consuming when the energy is close to the minimum. In the experiment for the horse model (Fig. 11) with $30 k$ vertices, the ICM entropy method takes several minutes, whereas the curvature diffusion method only takes $21 \mathrm{~s}$ to get the similar result. For other energy forms
(ABF, Area Distortion, U2), their gradients are more complicated than the one of curvature entropy energy; thus, they are computationally more expensive. Experiments show that the optimizations of curvature entropy energy and ABF energy lead to parameterizations with higher qualities than other 


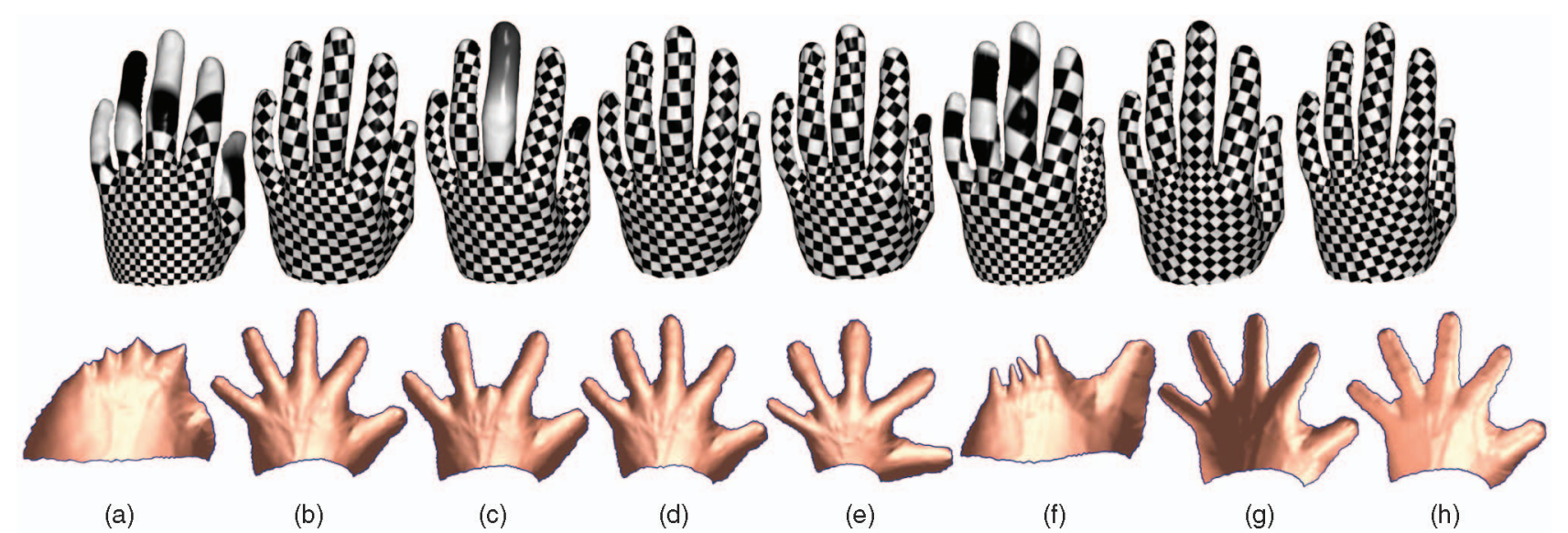

Fig. 10. Comparison of different parameterization with the Oliver's hand model: (a) initial, (b), (c), (d), and (e) optimizing ABF, AD, entropy, and U2, respectively, (f), (g), and (h) LSCM, circle patterns, and ABF++, respectively.

energies. The area distortion energy equation (6) has the local minima.

\section{Conclusion AND Future Work}

In this work, we introduce a set of rigorous theoretical tools and practical algorithms to solve the optimal conformal parameterization problem.

Inverse curvature map represents the exact analytical relation between area distortion and curvature as a dynamic Poisson system. This enables us to find the conformal parameterization with the least area distortion using nonlinear optimization techniques with linear constraints. The explicit conditions for the optima are deduced from the variational principle. A special energy form to measure the area distortion, called the curvature entropy, is investigated. It has a unique global minimum and can be optimized using curvature diffusion algorithm with free boundaries. Our experiments on complex meshes support our theoretical discoveries.

The inverse curvature map theorem is deduced for meshes with euclidean geometry, i.e., the mesh is formed by gluing euclidean triangles. We believe that the inverse curvature map holds for meshes with hyperbolic and spherical geometry, and leads to novel hyperbolic and spherical parameterization algorithms. Both of them play important roles for shape analysis and geometric modeling.

Although we solved the optimal parameterization problem for a given singular vertex set, it remains a challenging problem to determine the optimal vertex set. The common belief for choosing singular vertices is to pick the critical points of the area distortion function, as our algorithm in Section 3.3 does. In the future, we will apply our theoretic tools to continue the exploration along this direction.

\section{APPENDiX A}

\section{Proof of Inverse Curvature Map Theorem}

In this appendix, we give a detailed proof of the main theorem in this paper. The proof is based on analytic geometry. We have used Maple to derive various formulas. In order to save space, we omit the calculation results returned by Maple.
For a euclidean triangle $\left[v_{1}, v_{2}, v_{3}\right]$ with a circle packing metric with radii $\gamma_{1}, \gamma_{2}, \gamma_{3}$, and intersection angles $\phi_{12}, \phi_{23}$, $\phi_{31}$, the three common chords intersect in one point $O$ (see Fig. 3), called the radial center of three circles.

For a triangle with the circle packing metric, the following equations hold (the proof can be found in [24]):

$$
\frac{\partial \theta_{i}}{\partial u_{j}}=\frac{h_{k}}{l_{k}}
$$

where $u_{j}=\log \gamma_{j}$, and $h_{k}$ is the distance from the radial center $O$ to the edge $e_{k}$.

Also, we have (the proof can be found in [26] and [28]):

$$
\frac{\partial \theta_{i}}{\partial u_{j}}=\frac{\partial \theta_{j}}{\partial u_{i}} .
$$

Lemma 5.1. For a triangle with the circle packing metric, the derivative of $\theta_{i}$ satisfies

$$
d \theta_{i}=-\frac{h_{k}}{l_{k}}\left(d u_{i}-d u_{j}\right)-\frac{h_{j}}{l_{j}}\left(d u_{i}-d u_{k}\right) .
$$

Proof. Because the face is a euclidean triangle, $\theta_{i}+\theta_{j}+\theta_{k}=\pi$, and therefore, $\partial \theta_{i} / \partial u_{i}+\partial \theta_{j} / \partial u_{i}+\partial \theta_{k} / \partial u_{i}=0$. Because of symmetry in (10), $\partial \theta_{i} / \partial u_{i}=-\partial \theta_{i} / \partial u_{j}-\partial \theta_{i} / \partial u_{k}$. Therefore

$$
\begin{aligned}
d \theta_{i} & =\frac{\partial \theta_{i}}{\partial u_{i}} d u_{i}+\frac{\partial \theta_{i}}{\partial u_{j}} d u_{j}+\frac{\partial \theta_{i}}{\partial u_{k}} d u_{k} \\
& =-\frac{h_{k}}{l_{k}}\left(d u_{i}-d u_{j}\right)-\frac{h_{j}}{l_{j}}\left(d u_{i}-d u_{k}\right) .
\end{aligned}
$$

Now, we are ready to prove the main theorem:

Theorem 5.2 (inverse curvature map). The curvature map $K$ from a conformal class of circle packing metrics $\Pi_{\mathbf{u}}$ to the curvature space $\Omega_{\mathrm{k}}$ is a $C^{\infty}$ diffeomorphism. Furthermore, it is real analytic.

The derivative map $d K: T \Pi_{\mathbf{u}}(\mathbf{u}) \rightarrow T \Omega_{\mathbf{k}}(\mathbf{k})$ satisfies the discrete Poisson equation:

$$
d \mathbf{k}=\Delta(\mathbf{u}) d \mathbf{u},
$$




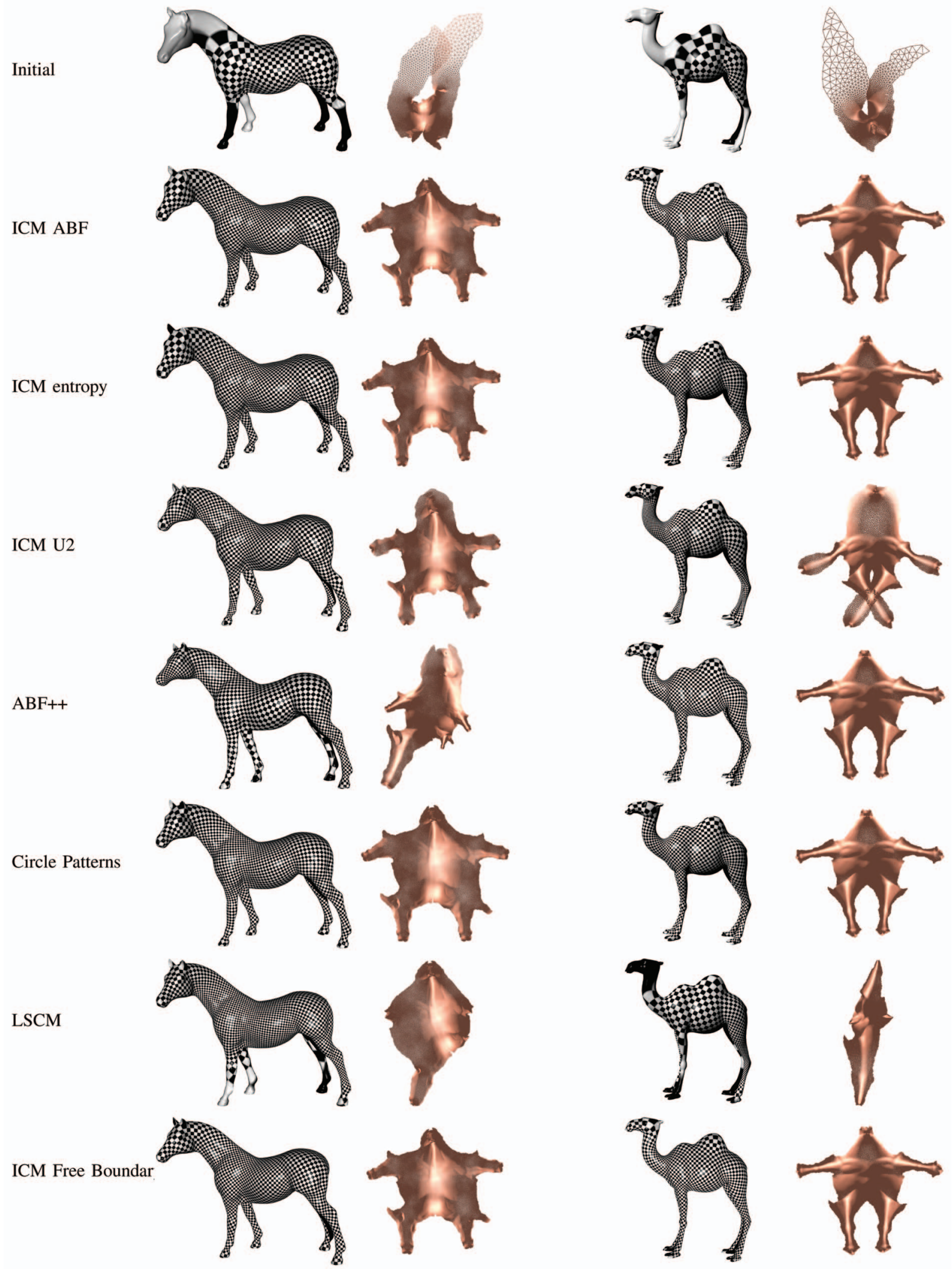

Fig. 11. Comparison.

where $T \Pi_{\mathbf{u}}(\mathbf{u})$ is the tangent space of $\Pi_{\mathbf{u}}$ at the point $\mathbf{u}$, $T \Omega_{\mathbf{k}}(\mathbf{k})$ is the tangent space of $\Omega_{\mathbf{k}}$ at the point $\mathbf{k}$, and $\Delta(\mathbf{u})$ is a positive definite matrix when constrained to $T \Pi_{\mathbf{u}}(\mathbf{u})$.
Proof. We consider the one-ring neighborhood of a vertex $v_{i}$. Let an adjacent face be $\left[v_{i}, v_{j}, v_{k}\right]$, where $\theta_{i}^{j k}$ denotes the angle at $v_{i}$ within the face. Then, from the definition of 


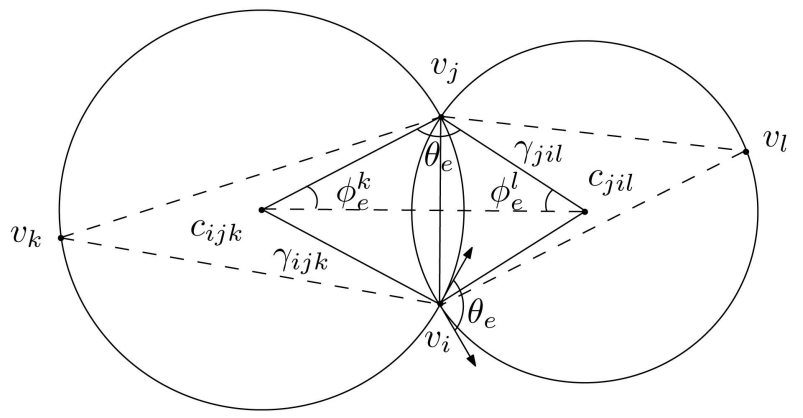

Fig. 12. Circle pattern metric.

discrete curvature and from (11) in Lemma 5.1, we get $d k_{i}=-\sum_{\left[v_{i}, v_{j}, v_{k}\right] \in F} d \theta_{i}^{j k}=\sum_{\left[v_{i}, v_{j}\right] \in E} w_{i j}\left(d u_{i}-d u_{j}\right)$, where $w_{i j}$ is the edge weight as defined in (9). If edge $\left[v_{i}, v_{j}\right]$ is adjacent to two faces $\left[v_{i}, v_{j}, v_{k}\right]$ and $\left[v_{j}, v_{i}, v_{l}\right]$, then its weight is equal to $w_{i j}=\frac{\partial \theta_{i}^{j k}}{\partial u_{j}}+\frac{\partial \theta_{i}^{j l}}{\partial u_{j}}$. In [24], Thurston gave a geometric proof to show that $\frac{\partial \theta_{i}}{\partial r_{j}}$ is positive if the radial center is inside the triangle, which is guaranteed if all edge angles $\phi_{i j}$ are acute. Therefore, all edge weights $w_{i j}$ are positive. In our work, we require all of the edge angles to be acute. The Jacobi matrix in (1) has the following characteristics: summation of each row is zero, and only the diagonal elements are positive. Using linear algebra, it can be shown that $J$ has a one-dimensional null space, spanned by $\mathbf{t}=(1, \cdots, 1)$, and $J$ is positive definite constrained on the complement space.

In the tangent space of admissible curvature space, because of the Gauss-Bonnet theorem, $d \mathbf{k}$ is orthogonal to $\mathbf{t}$, i.e., $\sum_{i} d k_{i}=0$. In the tangent space of the normalized conformal metric space, because of the normalization condition, $d \mathbf{u}$ is orthogonal to t, i.e., $\sum_{i} d u_{i}=0$. Therefore, the Jacobi matrix is invertible. According to the inverse function theorem, the curvature map $K$ : $\Pi_{\mathrm{u}} \rightarrow \Omega_{\mathrm{k}}$ is invertible.

By direct computation, the Jacobi matrix is differentiable to infinite degree, and so is its inverse. Therefore, the curvature map is a $C^{\infty}$ diffeomorphism. Furthermore, the explicit formula for the Jacobian shows that its elements are elementary functions of the $u_{i}$. Hence, the map is real analytic.

\section{APPENDIX B}

\section{Inverse Curvature Map Theorem in Circle Pattern Setting}

We used the notations in [30] for the following explanation. The configuration of circle pattern is shown in Fig. 12, two faces $\left[v_{i}, v_{j}, v_{k}\right]$ and $\left[v_{j}, v_{i}, v_{l}\right]$ sharing an edge $e=\left[v_{i}, v_{j}\right]$. The face circum-circles centered at $c_{i j k}$ and $c_{j i l}$ with radii $\gamma_{i j k}$ and $\gamma_{j i l}$, intersecting at an angle $\theta_{e}$. Let $\rho_{i j k}=\log \gamma_{i j k}, \rho_{j i l}=\log \gamma_{j i l}$, then

$$
\phi_{e}^{k}=\tan ^{-1} \frac{\sin \theta_{e}}{e^{x}-\cos \theta_{e}},
$$

where $x=\rho_{i j k}-\rho_{j i l}$. Then, the curvature of face $t \in T$ is defined as

$$
\Phi_{t}=2 \pi-\sum_{e \in t} 2 \phi_{e}^{t}
$$

Theorem 5.3 (inverse curvature map in circle pattern). Suppose $M$ is a closed mesh with a circle pattern. If the edge weights $\theta_{e} \in(0, \pi)$ are fixed, the face curvature map $\Phi$ : $\{\rho\} \rightarrow\left\{\Phi_{t}\right\}$ with the constraints $\sum_{t \in T} \rho_{t}=0$ is bijective and real analytic.

Proof. Similar to (9) and (10), direct computation shows

$$
\begin{aligned}
\frac{\partial \phi_{e}^{k}}{\partial \rho_{l}} & =\frac{\partial \phi_{e}^{l}}{\partial \rho_{k}} \\
& =\frac{\sin \theta_{e} e^{x}}{\left(e^{x}-\cos \theta_{e}\right)^{2}\left(1+\frac{\sin ^{2} \theta_{e}}{\left(e^{x}-\cos \theta_{e}\right)^{2}}\right)}>0 \text {, when } \theta_{e} \in(0, \pi) .
\end{aligned}
$$

Similar to Lemma 5.1, the following holds:

$$
d \phi_{e}^{k}=-\frac{\partial \phi_{e}^{k}}{\partial \rho_{l}}\left(d \rho_{k}-d \rho_{l}\right),
$$

then from (14), the Jacobian map is

$$
d \Phi=\Delta(\rho) d \rho,
$$

where $\Delta(\rho)$ has the same characteristics as (3). Therefore, it is positive definite.

The projected gradient optimization algorithm (Algorithm 4) and curvature diffuse algorithm (Algorithm 5) can be directly translated to the circle pattern setting.

\section{Appendix C}

\section{Embedding Induced by InVerse CuRvature MaP IS HARMONIC}

In this section, we give the formal proof to show that the embedding of the mesh induced by the inverse curvature map is harmonic. This builds the intrinsic connection to conventional harmonic maps.

Lemma 5.4. Suppose $M$ is a mesh with a circle packing metric, which induces zero Gaussian curvature everywhere, $f: M \rightarrow \mathbb{R}^{2}$ is the isometric embedding of $M$ onto the plane. Then, the embedding $f$ is harmonic, namely

$$
\Delta f=0 .
$$

Proof. As shown in Fig. 13, $v$ is a vertex on the mesh, $v_{0}, v_{1}, \cdots, v_{n-1}$ are the neighbor vertices of $v$, and $c_{i}$ is the center of the dual circle on the face $\left[v, v_{i}, v_{i+1}\right]$, which is orthogonal to the three circles centered at the vertices. We embed the one-ring neighborhood of $v$ onto the complex plane $\mathbb{C}$, and use the same symbol to represent the complex coordinates of the vertices. Then, by definition, the edge weight of edge $\left[v, v_{i}\right]$, denoted as $w_{i}$ equals to

$$
w_{i}=\frac{\left|c_{i-1} c_{i}\right|}{\left|v v_{i}\right|}, w_{i}\left(v_{i}-v\right)=\sqrt{-1}\left(c_{i}-c_{i-1}\right) .
$$




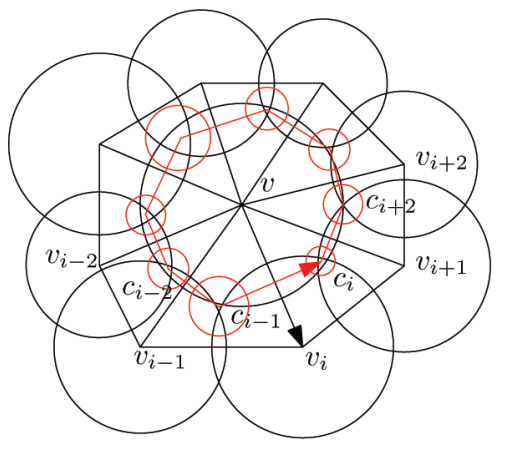

Fig. 13. The embedding induced by inverse curvature map is harmonic.

Therefore,

$$
\sum_{i} w_{i}\left(v_{i}-v\right)=\sum_{i} \sqrt{-1}\left(c_{i}-c_{i-1}\right)=0
$$

where this is equivalent to $\Delta f=0$. Hence, the coordinates of the vertices of $M$ are harmonic functions.

\section{ACKNOWLEDGMENTS}

The authors are grateful to Professor Tom Sederberg and Professor Ralph Martin for stimulating discussion. Special thanks to Professor Zhongxiao Jia in Tsinghua for his insightful feedback of the numerical computing problems. The authors thank Yukun Lai and Qianyi Zhou for their help for remeshing. The models used in this paper are the courtesy of Stanford University and AIM@SHAPE shape repository. This work is partially supported by the US National Science Foundation CCF-0448339, DMS-0626223, DMS-0528363, National Basic Research Project of China (Project 2006CB303102), the Natural Science Foundation of China (Project 60673004 and 60628202), and the National High Technology Research and Development Program of China (Project 2007AA01Z336). Feng Luo is partially supported by the NSF (DMS 0625935). Junho Kim is partially supported by the IITA\&MIC scholarship program.

\section{REFERENCES}

[1] M.S. Floater and K. Hormann, "Surface Parameterization: A Tutorial and Survey," Advances in Multiresolution for Geometric Modelling, Springer, pp. 157-186, 2005.

[2] A. Sheffer, E. Praun, and K. Rose, "Mesh Parameterization Methods and Their Applications," Foundations and Trends in Computer Graphics and Vision, vol. 2, Now Publisher, 2006.

[3] K. Hormann, B. Lévy, and A. Sheffer, "Mesh Parameterization: Theory and Practice," Proc. ACM SIGGRAPH, 2007.

[4] B. Lévy, S. Petitjean, N. Ray, and J. Maillot, "Least Squares Conformal Maps for Automatic Texture Atlas Generation," Proc. ACM SIGGRAPH '02, pp. 362-371, 2002.

[5] M. Desbrun, M. Meyer, and P. Alliez, "Intrinsic Parameterizations of Surface Meshes," Computer Graphics Forum, Proc. Eurographics '02, vol. 21, no. 3, pp. 209-218, 2002.

[6] M. Eck, T. DeRose, T. Duchamp, H. Hoppe, M. Lounsbery, and W. Stuetzle, "Multiresolution Analysis of Arbitrary Meshes," Proc. ACM SIGGRAPH, 1995.

[7] U. Pinkall and K. Polthier, "Computing Discrete Minimal Surfaces and Their Conjugates," Experimental Math., vol. 2, no. 1, pp. 15-36, 1993.
[8] M.S. Floater, "Parametrization and Smooth Approximation of Surface Triangulations," Computer Aided Geometric Design, vol. 14, no. 3, pp. 231-250, 1997.

[9] A. Sheffer and E. de Sturler, "Smoothing an Overlay Grid to Minimize Linear Distortion in Texture Mapping," ACM Trans. Graphics, vol. 21, no. 4, pp. 874-890, 2002.

[10] X. Gu and S.-T. Yau, "Global Conformal Parameterization," Proc. First Eurographics Symp. Geometry Processing (SGP '03), pp. 127-137, 2003.

[11] M.S. Floater, "Mean Value Coordinates," Computer Aided Geometric Design, vol. 20, no. 1, pp. 19-27, 2003.

[12] K. Hormann and G. Greiner, "Mips: An Efficient Global Parametrization Method," Curve and Surface Design: Saint-Malo 1999, pp. 153-162, Vanderbilt Univ. Press, 2000.

[13] P.V. Sander, J. Snyder, S.J. Gortler, and H. Hoppe, "Texture Mapping Progressive Meshes," Proc. ACM SIGGRAPH '01, pp. 409-416, 2001.

[14] O. Sorkine, D. Cohen-Or, R. Goldenthal, and D. Lischinski, "Bounded-Distortion Piecewise Mesh Parameterization," Proc. 13th IEEE Visualization Conf. (VIS '02), pp. 355-362, 2002.

[15] P. Degener, J. Meseth, and R. Klein, "An Adaptable Surface Parameterization Method," Proc. 12th Int'l Meshing Roundtable (IMR '03), pp. 201-213, 2003.

[16] S. Yoshizawa, A.G. Belyaev, and H.-P. Seidel, "A Fast and Simple Stretch-Minimizing Mesh Parameterization," Proc. IEEE Int'l Conf. Shape Modeling and Applications (SMI '04), pp. 200-208, 2004.

[17] E. Zhang, K. Mischaikow, and G. Turk, "Feature-Based Surface Parameterization and Texture Mapping," ACM Trans. Graphics, vol. 24, no. 1, pp. 1-27, 2005.

[18] Y. Lee, H.S. Kim, and S. Lee, "Mesh Parameterization with a Virtual Boundary," Computers and Graphics, vol. 26, no. 5, pp. 677-686, 2002.

[19] Z. Karni, C. Gotsman, and S.J. Gortler, "Free-Boundary Linear Parameterization of 3D Meshes in the Presence of Constraints," Proc. IEEE Int'l Conf. Shape Modeling and Applications (SMI '05), pp. 268-277, 2005.

[20] R. Zayer, C. Rössl, and H.-P. Seidel, "Setting the Boundary Free: A Composite Approach to Surface Parameterization," Proc. Third Eurographics Symp. Geometry Processing (SGP '05), H. Pottmann and M. Desbrun, eds., pp. 91-100, 2005.

[21] A. Sheffer and E. de Sturler, "Parameterization of Faceted Surfaces for Meshing Using Angle Based Flattening," Eng. Computers, vol. 17, no. 3, pp. 326-337, 2001.

[22] A. Sheffer, B. Lévy, M. Mogilnitsky, and A. Bogomyakov, "ABF++: Fast and Robust Angle Based Flattening," ACM Trans. Graphics, vol. 24, no. 2, pp. 311-330, 2005.

[23] R. Zayer, B. Lévy, and H.-P. Seidel, "Linear Angle Based Parameterization," Proc. Fifth Eurographics Symp. Geometry Processing (SGP '07), pp. 135-141, 2007.

[24] W.P. Thurston, Geometry and Topology of Three-Manifolds. Princeton Lecture Notes, 1976.

[25] B. Rodin and D. Sullivan, "The Convergence of Circle Packings to the Riemann Mapping," J. Differential Geometry, vol. 26, no. 2, pp. 349-360, 1987.

[26] C.R. Collins and K. Stephenson, "A Circle Packing Algorithm," Computational Geometry: Theory and Applications, vol. 25, pp. 233-256, 2003.

[27] R.S. Hamilton, "The Ricci Flow on Surfaces," Math. and General Relativity, vol. 71, pp. 237-262, 1988.

[28] B. Chow and F. Luo, "Combinatorial Ricci Flows on Surfaces," J. Differential Geometry, vol. 63, no. 1, pp. 97-129, 2003.

[29] M. Jin, F. Luo, and X. Gu, "Computing Surface Hyperbolic Structure and Real Projective Structure," Proc. ACM Symp. Solid and Physics Modeling (SPM '06), pp. 105-116, 2006.

[30] L. Kharevych, B. Springborn, and P. Schröder, "Discrete Conformal Mappings via Circle Patterns," ACM Trans. Graphics, vol. 25, no. 2, pp. 412-438, 2006.

[31] A.I. Bobenko and B.A. Springborn, "Variational Principles for Circle Patterns and Koebe's Theorem," Trans. Am. Math. Soc., vol. 356, pp. 659-689, 2004.

[32] M. Ben-Chen, C. Gotsman, and G. Bunin, "Conformal Flattening by Curvature Prescription and Metric Scaling," Computer Graphics Forum, Proc. Eurographics '08, vol. 27, no. 2, to appear.

[33] B.A. Springborn, "Variational Principles for Circle Patterns," PhD thesis, Technical Univ. of Berlin, 2003.

[34] F. Luo, X. Gu, and J. Dai, Variational Principles for Discrete Surfaces. High Education Press, 2007. 
[35] A.P. Witkin and P.S. Heckbert, "Using Particles to Sample and Control Implicit Surfaces," Proc. ACM SIGGRAPH'94, pp. 269-277, 1994.

[36] Y.-K. Lai, Q.-Y. Zhou, S.-M. Hu, J. Wallner, and H. Pottman, "Robust Feature Classification and Editing," IEEE Trans. Visualization and Computer Graphics, vol. 13, pp. 34-45, 2007.

[37] N. Ray, W.C. Li, B. Lévy, A. Sheffer, and P. Alliez, "Periodic Global Parameterization," ACM Trans. Graphics, vol. 25, no. 4, pp. 1460-1485, 2006.

[38] J. Nocedal and S.J. Wright, Numerical Optimization. Springer, 1999.

[39] X. Gu, S.J. Gortler, and H. Hoppe, "Geometry Images," ACM Trans. Graphics, vol. 21, no. 3, pp. 355-361, 2002.

[40] A. Sheffer and J.C. Hart, "Seamster: Inconspicuous LowDistortion Texture Seam Layout," Proc. 13th IEEE Visualization Conf. (VIS), 2002

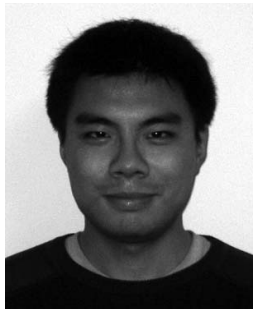

Yong-Liang Yang received the bachelor's degree in computer science from Tsinghua University, Beijing, in 2004. He is a PhD student in the Department of Computer Science and Technology, Tsinghua University. His research interests include computer graphics, geometric modeling, and processing.

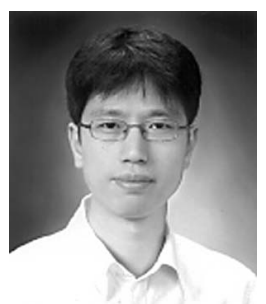

Junho Kim received the $\mathrm{BS}, \mathrm{MS}$, and $\mathrm{PhD}$ degrees in computer science and engineering from Pohang University of Science and Technology (POSTECH), in 1998, 2000, and 2005, respectively. In his $\mathrm{PhD}$ course, he worked at RWTH Aachen University as a BK21 visiting $\mathrm{PhD}$ student from 2003 to 2004 . After getting his $\mathrm{PhD}$ degree, he worked at Stony Brook University, Stony Brook, New York, as a research associate from 2005 to 2008 . He is currently a full-time lecturer of game engineering in the College of Visual Image and Information Technology, Dong-Eui University, Busan, Korea. His research interests include computer graphics, computer vision, geometric modeling, and interactive games.

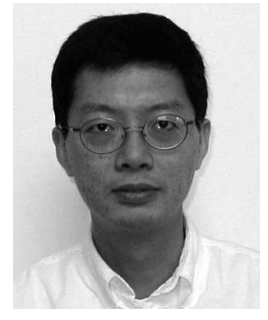

Feng Luo received the $\mathrm{PhD}$ degree from the University of California, San Diego, in 1989. $\mathrm{He}$ is a professor of mathematics in the Department of Mathematics, Rutgers University, Piscataway, New Jersey. His research interests include geometry and topology on surfaces and 3-manifolds, and computer graphics. For more information, see http:// www. math. rutgers.edu/ fluo.

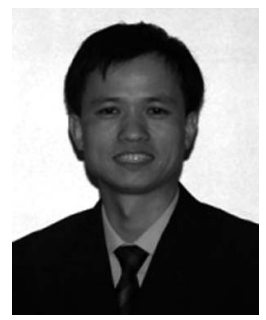

Shi-Min Hu received the $\mathrm{PhD}$ degree from Zhejiang University, in 1996. He is currently a chair professor of computer science in the Department of Computer Science and Technology, Tsinghua University, Beijing. His research interests include digital geometry processing, video processing, rendering, computer animation, and computer-aided geometric design. $\mathrm{He}$ is on the editorial boards of Computer Aided Design. He is a member of the IEEE.

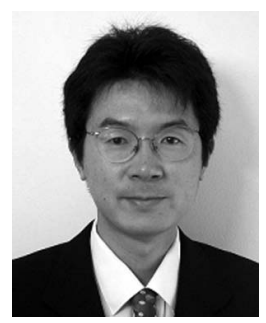

Xianfeng $\mathrm{Gu}$ received the $\mathrm{PhD}$ degree in computer science from Harvard University, in 2003. He is an assistant professor of computer science at Stony Brook University, Stony Brook, New York. He won the US National Science Foundation CAREER award in 2004. His research interests include computer graphics, computer vision, and medical imaging. His major works include geometry images, global conformal surface parameterization, manifold splines, and computational conformal geometry. For more information, see http://www.cs.sunysb.edu/ gu. He is a member of the IEEE and the IEEE Computer Society.

$\triangleright$ For more information on this or any other computing topic, please visit our Digital Library at www.computer.org/publications/dlib. 